Matemática Aplicada, Computacional e Industrial

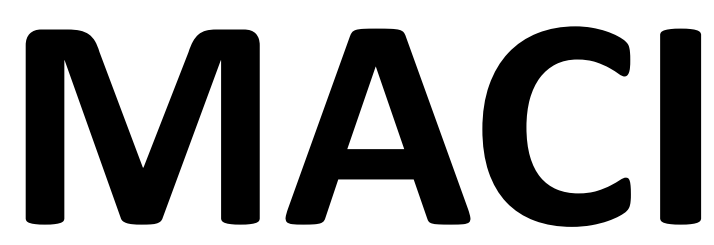

Vol. 8

2021

Trabajos presentados al VIII MACI 2021

Proceedings of VIII MACI 2021

La Plata, 3 al 7 de mayo de 2021

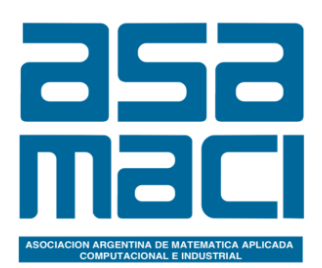




\section{MATEMÁTICA APLICADA, COMPUTACIONAL E INDUSTRIAL}

ISSN: $2314-3282$

\section{Directora}

Cristina Maciel, Universidad Nacional del Sur, Bahía Blanca

\section{Comité Editorial}

Carlos D'Attellis, Universidad Favaloro - UNSAM, Buenos Aires

Pablo Jacovkis, UBA, UNTreF, Buenos Aires

Sergio Preidikman, CONICET - UNC, Córdoba

Diana Rubio, UNSAM, Buenos Aires

Juan Santos, CONICET - IGP - UBA, Buenos Aires

Rubén Spies, IMAL- CONICET - UNL, Santa Fe

Domingo Tarzia, CONICET-UA, Rosario

Cristina Turner, CONICET -UNC, Córdoba

\section{Volumen 8, 2021}

Contiene los trabajos presentados al congreso VIII MACI 2021, La Plata, Argentina.

\section{Editores}

María Laura Schuverdt, CONICET - UNLP, La Plata

Nadia Kudraszow, CONICET - UNLP, La Plata

Raúl P. Vignau, UNLP, La Plata

María Daniela Sánchez, UNLP, La Plata

\section{ASAMACI}

Asociación Argentina de Matemática Aplicada, Computacional e Industrial

Güemes 3450, (3000) Santa Fe, Argentina

E-mail: asamaci@gmail.com

http://asamaci.org.ar/

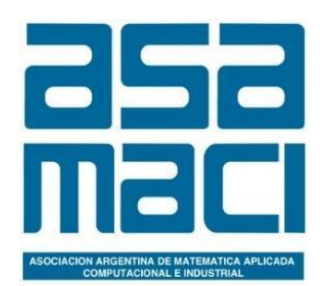




\section{PREFACIO}

El presente volumen contiene los trabajos aceptados en el VIII Congreso de Matemática Aplicada, Computacional e Industrial (VIII MACI - 2021), realizado de forma virtual entre los días 3 y 7 de mayo de 2021 organizado en la ciudad de La Plata, Argentina.

El congreso bianual MACI convoca a la comunidad de profesionales del área de la Matemática Aplicada, Computacional e Industrial, de Argentina y la región. Organizado por ASAMACI y AR-SIAM, cada realización tiene lugar en una ciudad diferente de Argentina, habiéndose realizado los anteriores en Córdoba (2007), Rosario (2009), Bahía Blanca (2011), Buenos Aires (2013), Tandil (2015), Comodoro Rivadavia (2017) y Río Cuarto (2019).

Estas reuniones tienen como objetivo la difusión de trabajos originales o en desarrollo, el planteo de problemáticas abiertas, el fomento de la investigación y motivación para la formación de recursos humanos en las distintas disciplinas que se desarrollan dentro de la Matemática Aplicada, Computacional e Industrial.

Estas actividades se enmarcan en las políticas generales de la Sección Argentina de la Society for Industrial and Applied Mathematics (AR-SIAM) y la Asociación Argentina de Matemática Aplicada Computacional e Industrial (ASAMACI), creadas por iniciativa de profesionales locales en 2006 y 2008 , respectivamente. Cabe destacar, que la presente edición, se realizó en homenaje a Domingo Tarzia en el año de su septuagésimo aniversario.

En esta octava edición se aceptaron para su publicación 178 trabajos, los cuales fueron expuestos en forma oral durante el congreso. Los mismos fueron previamente evaluados por revisores anónimos y, consecuentemente, revisados por los autores en una segunda instancia. Gracias a esto muchos de los manuscritos fueron sustancialmente mejorados, en calidad y originalidad, en base a las sugerencias de los revisores. Los trabajos, según su temática, se enmarcaron en veintiséis sesiones.

Durante el Congreso se dictaron siete conferencias plenarias a cargo de los profesores Cleve Moler (MathWorks Inc.), Mabel Tidball (INRAE-Montpellier, Francia), Claudia Pons (Universidad Abierta Interamericana UAI y UNLP, Argentina), Mikhail Solodov (IMPA Instituto de Matemática Pura e Aplicada, Brasil), Claudia Sagastizábal (Unicamp, Brasil), Inés Armendáriz (Universidad de Buenos Aires, Argentina) y Enrique Pujals (City University of New York, EEUU). Se ofrecieron cuatro cursos para estudiantes avanzados de grado y de posgrado, impartidos por los profesores Marcos Raydan (Universidad NOVA de Lisboa, Portugal), Paula Zabala (Universidad de Buenos Aires, Argentina), Horacio Rotstein (New Jersey Institute of Technology, Rutgers University, EEUU) y Esteban Tabak (New York University, EEUU).

Además, se realizaron tres mesas redondas abiertas a todo público:

* "Matemática Aplicada en la Industria y Sociedad", coordinada por Damián Fernández y Lisandro Parente, la cual contó con los siguientes invitados: Carlos Pita (JAMPP, Argentina), Matías Lee, María Martínez y Francisco Cucullu (Xcapit, Argentina), Juan Carlos de los Reyes (MODEMAT, Escuela Politécnica Nacional, Ecuador) y Gonzalo Pita (Johns Hopkins Whiting School of Engineering, EEUU).

* "La Matemática del COVID", coordinada por Claudia Gariboldi, la cual contó con los siguientes invitados: Juan Pablo Aparicio (INENCO-UNSa, Argentina), Guillermo Durán (CONICET-UBA, Argentina), Pablo Lotito (PLADEMA-UNCPBA, Argentina) y Claudia Sagastizábal (Unicamp, Brasil).

* "Matemática Aplicada en la Educación", coordinada por Mabel Rodríguez y Gabriel Soto, la cual contó con los siguientes invitados: César Retamal (Univ. de Talca, Chile), Marcel Pochulu (Univ. Nac. de Villa María, Argentina), Flavio Guiñez (Univ. de Chile, Chile), Pablo Carranza (Univ. Nac. de Río Negro, Argentina). 

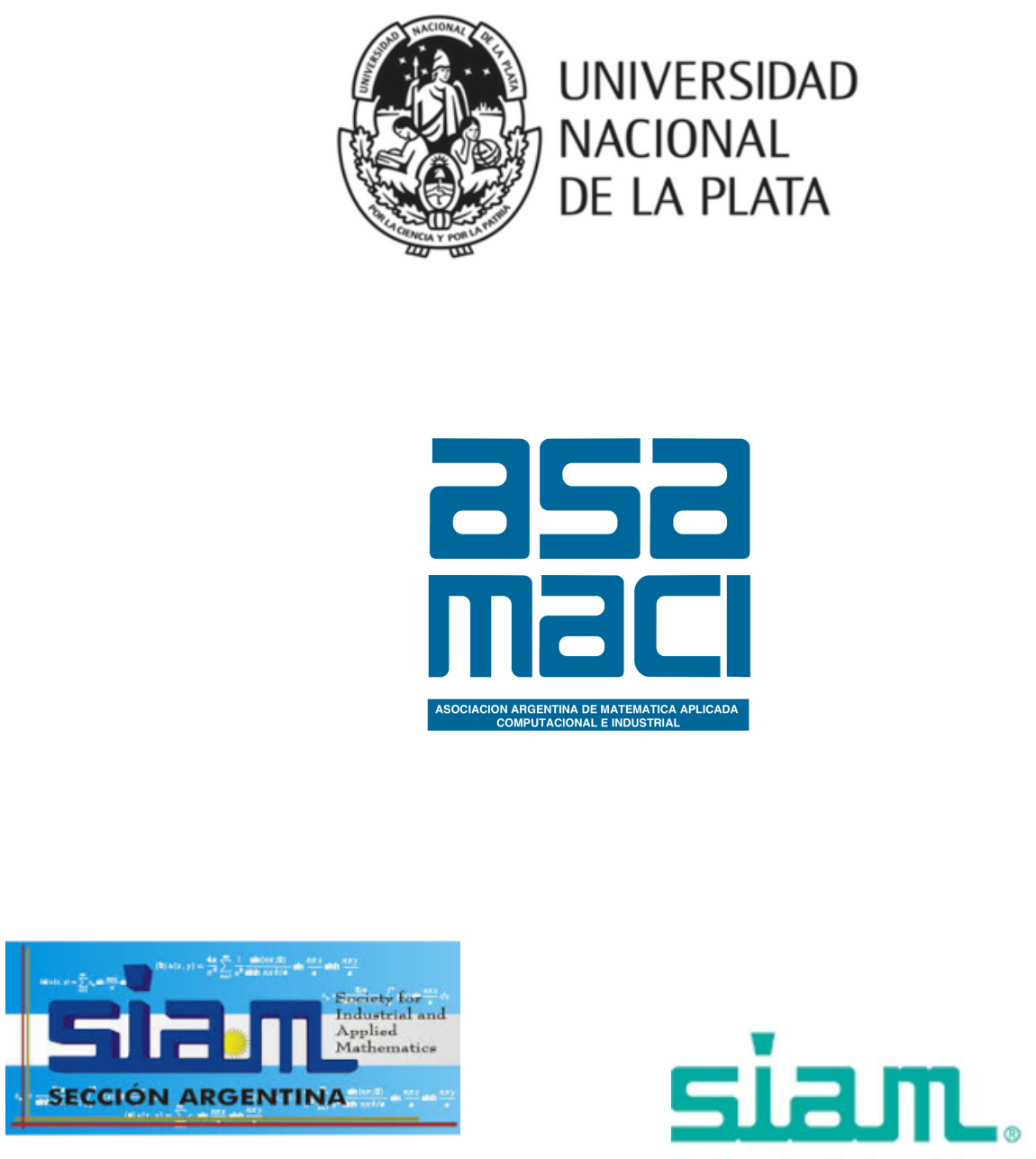

Society for Industrial and Applied Mathematics

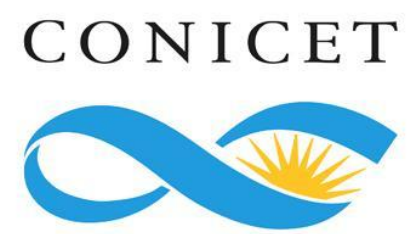

Consejo Nacional de Investigaciones 


\section{COMITÉ CIENTÍFICO}

Pablo Jacovkis (UNTREF-UBA, Ar).

Pablo A. Lotito (PLADEMA-UNCPBA, Ar).

Cristina Maciel (UNS, Ar).

Diana Rubio (UNSAM, Ar).

Claudia A. Sagastizábal (ICMC-USP, Br).

Ma. Laura Schuverdt (UNLP, Ar).

Rubén D. Spies (IMAL CONICET-UNL, Ar)

\section{COMITÉ ORGANIZADOR LOCAL}

\section{Coordinadores Generales:}

Ma. Laura Schuverdt

Raúl P. Vignau

Ma. Daniela Sánchez

Nadia L. Kudraszow

\section{Colaboradores:}

Eduardo Chiumiento (UNLP, Ar)

María del Rosario Etchechoury (UNLP, Ar)

M. Victoria Fasano (UNLP, Ar)

Blas Fernández (UNLP, Ar)

Alejandra Garrido (UNLP, Ar)

Noemí Gudiño (UNLP, Ar)

Diana Kleiman (UNLP, Ar)

Noemí Lubomirsky (UNLP, Ar)

Victoria Vampa (UNLP, Ar)

Marcela Zuccalli (UNLP, Ar) 


\section{SESIONES Y COORDINADORES}

Sesión Plenaria en honor a Domingo Tarzia - Sabrina Roscani

1. Análisis Matricial - Néstor Thome Coppo, Fabián Levis

2. Análisis Numérico - Ariel Lombardi, Alberto Ferrari

3. Computación de alto desempeño - Juan Pablo D’Amato, Pablo Rinaldi

4. Control y Control Óptimo - Lisandro Parente, Justina Gianatti

5. Ecuaciones Diferenciales - Mariano de Leo, Damián Knopoff

6. Geometría Diferencial - Carlos Olmos, Julio Barros, Bruno Roccia

7. Investigación Operativa - Javier Marenco, Ivo Koch

8. Optimización - Cristina Maciel, María de Gracia Mendonça

9. Probabilidad y Estadística - Beatriz Marrón, Marcelo Ruiz

10. Problemas de Frontera Libre - Claudia Lederman, Sabrina Roscani

11. Problemas Inversos - María Inés Troparevsky, Silvia Alejandra Seminara

12. Sistemas Dinámicos - Guillermo La Mura, Marcela Fabio

13. Biomatemática - Mercedes Pérez Millán, Gabriel Soto

14. Ciencia de Datos y Aprendizaje Automático - Pablo Lotito

15. Economía Matemática - Pablo Neme, Agustin Bonifacio

16. Finanzas Cuantitativas - Gabriel Basaluzzo, Manuel Maurette

17. Matemática Industrial - Miguel Cavaliere

18. Mecánica Computacional - : Martín Pucheta, Santiago Márquez Damian

19. Mecánica del Continuo - Sergio Preidikman, Sergio Elaskar

20. Modelos Matemáticos Interdisciplinarios - Pablo Jacovkis

21. Señales e Imágenes - Susana Ferrero, Juan Fontana

22. Transferencia de Calor y Materia - Demian Goos

23. Visión Computacional - Mariana del Fresno, José Massa

24. Aportes matemáticos frente al Covid-19 - Pablo Lotito

25. Pósteres de Estudiantes - Marcela Fabio, Marcela Morvidone 


\section{ÍNDICE}

SESIÓN PLENARIA EN HONOR A DOMINGO TARZIA .1

ELLIPTIC QUASI-VARIATIONAL-HEMIVARIATIONAL INEQUALITIES WITH APPLICATION

S. Migórski y S. Dudek 3

ESTIMACIÓN DE PARÁMETROS EN MATERIALES COMPUESTOS PARA EL ANÁLISIS DE LA CORROSIÓN PROVOCADA POR ALTAS TEMPERATURAS

D. Rubio, M. Morvidone y N. Saintier . .7

IMPLICIT SWEEPING PROCESS ARGUMENTS IN CONTACT MECHANICS

M. Sofonea 11

A MATHEMATICAL MODEL FOR TUMOR ANGIOGENESIS WITH A TRAVELLING WAVE STRUCTURE

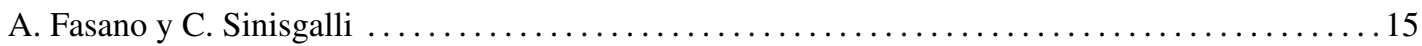

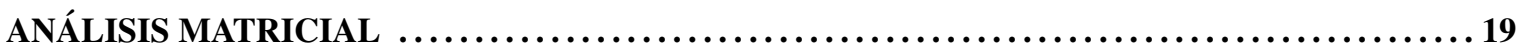

CÁLCULO DE LAS INVERSAS GD Y GDMP

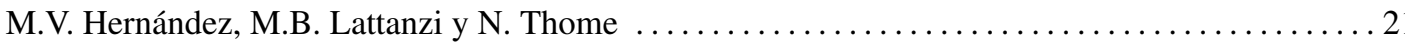

SOBRE UNA CLASE ESPECIAL DE INVERSAS EXTERIORES

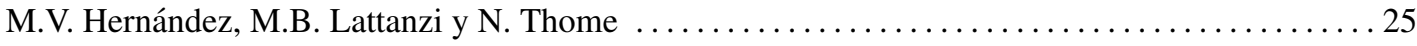

CONMUTATIVIDAD DE MATRICES CON SUS INVERSAS GENERALIZADAS

D.E. Ferreyra, F.E. Levis, A.N. Priori y N. Thome ….......................... 29

SOBRE EL ORDEN PARCIAL GRUPO PARA MATRICES COMPLEJAS

L. Rueda y N. Thome .33

LA INVERSA GENERALIZADA BT DE UNA MATRIZ RECTANGULAR

D.E. Ferreyra, C. Torigino y N. Thome

SPECIAL PROPERTIES OF TIME EVOLUTION OF STOCHASTIC MATRICES AND THEIR APPLICATION IN TRAFFIC OPTIMIZATION

S.S. Mizrahi, D. Otero y L. Robles Dávila $\ldots \ldots \ldots \ldots \ldots \ldots \ldots \ldots \ldots \ldots \ldots \ldots \ldots \ldots \ldots \ldots \ldots \ldots$

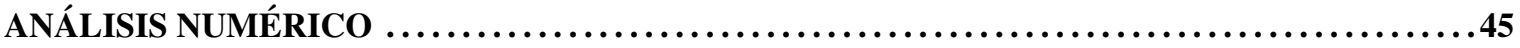

FINITE ELEMENT APPROXIMATION OF FRACTIONAL NEUMANN PROBLEMS

F.M. Bersetche y J.P. Borthagaray

NUMERICAL APPROACH TO SECOND-ORDER FRACTIONAL DIFFERENTIAL EQUATIONS

G. Monzón

APLICACIÓN DE DOS MÉTODOS NUMÉRICOS A UN MODELO DE ORDEN FRACCIONARIO PARA EL TRATAMIENTO DEL VIH

A.J. Ferrari, L.P. Lara, M.C. Olguin y E.A. Santillan Marcus 55 
QUASI-INTERPOLATION IN SPLINE SPACES: LOCAL STABILITY AND APPROXIMATION PROPERTIES

M.E. Castillo y E.M. Garau 59

APROXIMACIÓN MEDIANTE ELEMENTOS FINITOS MIXTOS DEL PROBLEMA DE STOKESDARCY EN DOMINIOS CURVOS

M.G. Armentano y M.L. Stockdale .63

MODELADO DE CORRIENTES DE MAREAS EN ZONAS COSTERAS APLICADO AL GOLFO SAN JORGE

I. Mandelman, M. Ferrari y D. Fernández .67

POTENCIAL DE COULOMB PARA FUNCIONES 1S DE SLATER EXPRESADO EN TÉRMINOS DE FUNCIONES DE BESSEL

C.J. Alturria Lanzardo, J.E. Pérez, M.L. Tardivo, G. Fraschetti y J.C. Cesco .71

A PRIORI ERROR ANALYSIS FOR UNUSUAL LOCAL DISCONTINUOUS GALERKIN

T.P. Barrios y R. Bustinza 75 AN A POSTERIORI ERROR ANALYSIS FOR A DUAL MIXED FORMULATION OF THE NON HOMOGENEOUS DIRICHLET PROBLEM

T.P. Barrios, R. Bustinza y C. Campos .79

RESOLUCIÓN EFECTIVA DE ECUACIONES ELÍPTICAS NO LINEALES CON CRECIMIENTO NO ESTÁNDAR EN 2D

A. Aragón, J. Fernández Bonder y D. Rubio 83

A DEEP FIRST-ORDER SYSTEM LEAST SQUARES METHOD FOR SOLVING ELLIPTIC PDES

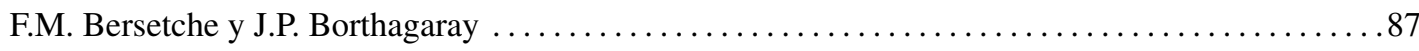

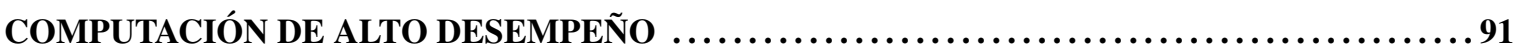

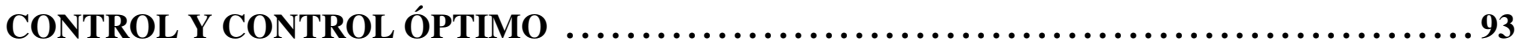

CONTROL DE PYRAGAS PARA ESTABILIZAR TRAYECTORIAS. SINCRONIZACIÓN EN TORNO A UN EQUILIBRIO INESTABLE

C.H.D. Alliera 95

ANÁLISIS DE UN MODELO MATEMÁTICO EN ECUACIONES DIFERENCIALES ORDINARIAS APLICADO A LA TRASMISIÓN DEL VIH EN LA CIUDAD DE PASTO-NARIÑO, CONSIDERANDO ESTRATEGIAS DE PROTECCIÓN

M.N. Guerrero Laos y M.O. Cerón Gomez .99

CONTROL ÓPTIMO FRONTERA NEUMANN PARA ECUACIONES VARIACIONALES PARABÓLICAS Y ELÍPTICAS

C.M. Bollo, C.M. Gariboldi y D.A. Tarzia

CONTROL ÓPTIMO PARA UNA INECUACIÓN CUASIVARIACIONAL DIFERENCIAL

J. Bollati, M. Sofonea y D.A. Tarzia 107 
EXISTENCIA DE EQUILIBRIO PARA UN PROBLEMA DE JUEGOS A CAMPO MEDIO DE CONTROLES CON RESTRICCIONES

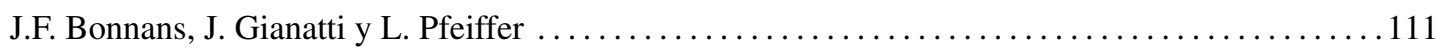

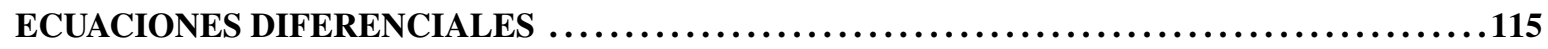

FORMULACIÓN DEL PROBLEMA DE CAUCHY Y DE CONDICIONES DE BORDE PARA UN SISTEMA DE ECUACIONES HIPERBÓLICAS DE EVOLUCIÓN

J.C. Barreto y J.L. Mroginski

CIRCUITOS ELÉCTRICOS: SINGULARIDADES Y PUNTOS HIT

D. Kleiman, V. Vampa, C. González y M. Etchechoury

ACCURATE SOLUTIONS OF THE THOMAS-FERMI EQUATION USING THE HANKEL-PADÉ METHOD

F.M. Fernández y J. Garcia

SPECTRAL DECOMPOSITION FOR THE 1-D SCHRÖDINGER-POISSON EQUATION

N. Biedma y M. De Leo

ECUACIÓN DE GINZBURG LANDAU COMPLEJA CON UN TÉRMINO POTENCIAL EN ESPACIOS DE ZHIDKOV

A. Besteiro

EXISTENCIA, COMPARACIÓN, Y CONVERGENCIA PARA UNA CLASE DE INECUACIONES HEMIVARIACIONALES ELÍPTICAS.

C.M. Gariboldi, S. Migórski, A. Ochal y D.A. Tarzia

SOBRE LAS SOLUCIONES DE UNA FAMILIA DE PROBLEMAS DE VALORES INICIALES Y DE CONTORNO DE TIPO ROBIN PARA LA ECUACIÓN DE DIFUSIÓN FRACCIONARIA EN EL TIEM$\mathrm{PO}$

I. Cardoso, S. Roscani y D. Tarzia

OPINION FORMATION MODEL IN A HIERARCHICAL SOCIETY

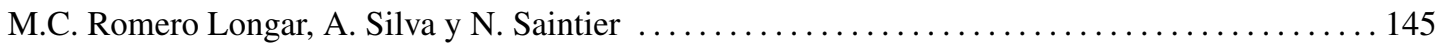

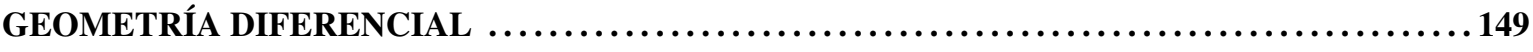

CURVAS EN SUPERFICIES DE REVOLUCIÓN CON CURVATURA GAUSSIANA CONSTANTE

L.M. Mareco Franco y J.C. Barros

LA ENERGÍA DE LAS DISPOSICIONES DE SUBESPACIOS AFINES A DISTANCIA UNITARIA DEL ORIGEN

F. Ferraris, R.P. Moas y M. Salvai

SUPERFICIES CUÁDRICAS PARALELAS

J.M. Ocampos Monges y J.C. Barros

REDUCTION BY SYMMETRIES AND HIGHER-ORDER MAGNETIC SYSTEMS

L. Colombo, M.E. Eyrea Irazú y M. Zuccalli 
PROBLEMAS DE RUTEO DE VEHÍCULOS CON CONFLICTOS POR VÉRTICES

D. Delle Donne, I. Koch y S. Montiel 169

EL VERTIDO ÓPTIMO DE ENERGÍA RENOVABLE EN LA OPERACIÓN ELÉCTRICA DEL SISTEMA INTERCONECTADO

R. Di Pasquale y J. Marenco

A BRANCH-AND-CUT ALGORITHM FOR THE ROUTING AND SPECTRUM ALLOCATION PROBLEM

M. Bianchetti y J. Marenco

SOBRE LA CLIQUE COLORACIÓN DE LOS GRAFOS $[4,2,2]$

P.J. De Caria, M.P. Mazzoleni y M.G. Payo Vidal

ON CONTACT $B_{0}$-VPG GRAPHS WITH BOUNDED NUMBER OF HOLES

F. Bonomo-Braberman, E. Galby, C.L. Gonzalez y M.P. Mazzoleni ....................... 185

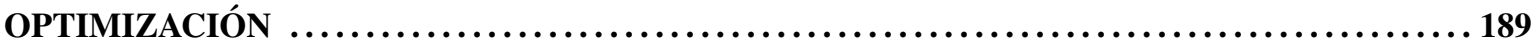

DOS ESTRATEGIAS NO MONÓTONAS DE REGIÓN DE CONFIANZA PARA UN PROBLEMA MULTIOBJETIVO

V.A. Ramirez y G.N. Sottosanto 191

COMPARACIÓN DE TRES MÉTODOS PARA PROBLEMAS DE OPTIMIZACIÓN MULTIOBJETIVO NO CONVEXOS

G.A. Carrizo

A NONMONOTONE FILTER ALGORITHM FOR UNCONSTRAINED MULTIOBJECTIVE OPTIMIZATION BASED ON TRUST REGION

M.G. Mendonça

UNA CONDICIÓN DE CALIDAD DE SEGUNDO ORDEN BASADA EN CURVAS

N.S. Fazzio, M.D. Sánchez y M.L. Schuverdt

OPTIMALITY CONDITIONS AND GLOBAL CONVERGENCE FOR NONLINEAR SEMIDEFINITE PROGRAMMING

R. Andreani, G. Haeser y D. Santos

CUMPLIMIENTO DE UNA CONDICIÓN NECESARIA DE OPTIMALIDAD DE SEGUNDO ORDEN

D. Fernández

UNCERTAIN FARTHEST VORONOI CELLS

A.B. Ridolfi y V.N. Vera de Serio

OPTIMIZACIÓN APLICADA AL DISEÑO DE MATERIALES POROSOS CON MICROESTRUCTURA PERIÓDICA

J.L. Mroginski, J.M. Podestá, H.A. Di Rado y J.C. Barreto 
OPTIMIZACIÓN DE DESLASTRE DE CARGAS EN REDES DE DISTRIBUCIÓN UTILIZANDO PSO

L.E. Valenzuela y A. Rubiales

ON THE GEOMETRIC SOLUTION OF STEINER PROBLEMS USING LEVEL BUNDLE METHODS

J.C. Medeiros y S.A. Santos

CONVERGENCIA DEL MÉTODO DEL LAGRANGIANO AUMENTADO NO DIFERENCIABLE USANDO SUAVIZADO

J.L. Romero y D. Fernández

UN ALGORITMO INEXACTO PARA INECUACIONES VARIACIONALES ESTOCÁSTICAS MULTIETAPA

E.L. Buscaglia, P.A Lotito y L.A. Parente

PROBABILIDAD Y ESTADÍSTICA

INFLUENCIA ESPACIAL DEL NIVEL DE SIGNIFICANCIA EN EL ANÁLISIS DE TENDENCIA DE LAS PRECIPITACIONES ANUALES EN LA ARGENTINA

J.F. Weber

ACEPTACIÓN DE UN SELLO DE CALIDAD HORTÍCOLA. UNA APLICACIÓN DE TABLAS DE CONTINGENCIA A TRES VÍAS

N. Vellini y B. Lupín

MEDIDA DE DISTANCIA A UNA DISTRIBUCIÓN DE PROBABILIDAD MODELO

M.A. Ré

EVALUACIÓN DE MÉTODOS DE SELECCIÓN DE COVARIANZA EN ALTA DIMENSIÓN

J.R. Maldonado y M. Ruiz

RANDOM SAMPLING OVER LCA GROUPS AND INVERSION OF THE RADON TRANSFORM

E. Porten, J.M. Medina y M. Morvidone .................................... 257

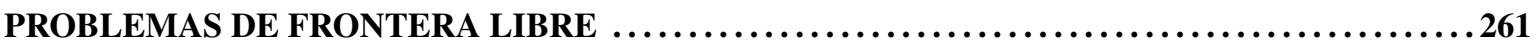

REGULARITY THEORY FOR A CLASS OF FULLY NONLINEAR FREE TRANSMISSION PROBLEMS

E.A. Pimentel y M.S. Santos

UN PROBLEMA DE AUTOVALORES DE STEKLOV CON RESTRICCIÓN DE VOLUMEN

A. Salort, B. Schvager y A. Silva 265

ONE-PHASE FREE BOUNDARY PROBLEMS WITH NON HOMOGENEOUS DEGENERACY: REGULARITY OF SOLUTIONS AND FREE BOUNDARIES

J.V. da Silva, G.C. Rampasso, G.C. Ricarte y H.A. Vivas 269

UN PROBLEMA DE STEFAN A UNA FASE PARA LA ECUACIÓN DE CONVECCIÓN-DIFUSIÓN CON UNA FUENTE DE CALOR

J. Bollati y A.C. Briozzo

A BIASED REVIEW OF SPACE-FRACTIONAL DIFFUSION PROBLEMS

P. Rybka 
COMPACTNESS AND DICHOTOMY IN NONLOCAL SHAPE OPTIMIZATION

E. Parini y A.M. Salort 281

AN OPTIMIZATION PROBLEM WITH VOLUME CONSTRAINT FOR THE INHOMOGENEOUS $p(x)$-LAPLACIAN

C. Lederman y N. Wolanski

SOLUCIONES AUTO-SIMILARES PARA DOS PROBLEMAS DE STEFAN FRACCIONARIOS EN EL ESPACIO

S.D. Roscani, L.D. Venturato y D.A. Tarzia 289

A DIFFUSIVE INTERFACE MODEL FOR TIME FRACTIONAL SOLID TO LIQUID PHASE CHANGE

V.R. Voller

SINGULARLY PERTURBED MODELS WITH NON-HOMOGENEOUS DEGENERACY

J.V. da Silva, E.C.B. Júnior y G.C. Ricarte

ABOUT THE EXISTENCE OF AN ALT-CAFFARELLI-FRIEDMAN MONOTONICITY FORMULA IN THE HEISENBERG GROUP

F. Ferrari y N. Forcillo

EXISTENCIA Y UNICIDAD DE SOLUCIÓN DE UN PROBLEMA DE STEFAN A UNA FASE CON UNA FUENTE DE CALOR QUE DEPENDE DEL FLUJO DE TEMPERATURA EN EL BORDE FIJO

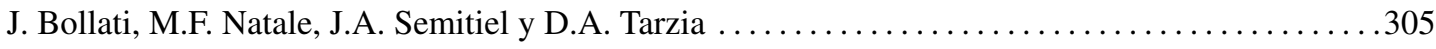

NON-CONVEX HAMILTON-JACOBI EQUATIONS WITH GRADIENT CONSTRAINTS

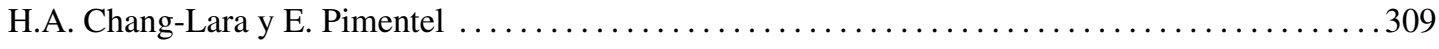

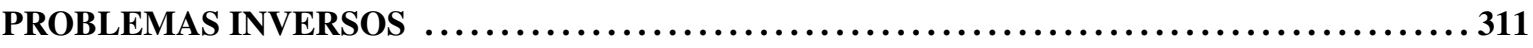

ERROR ESTIMATES FOR DOUBLY-GENERALIZED TIKHONOV-PHILLIPS REGULARIZATION WITH MULTIPLE PENALIZERS

M.J. Carrió, G.L. Mazzieri y K.G. Temperini

RECOVERING THE INITIAL CONDITION OF PARABOLIC EQUATIONS FROM LATERAL CAUCHY DATA AS A GENERALIZED PROBLEM OF MOMENTS

M.B. Pintarelli

AN INVERSE SOURCE PROBLEM FOR THE FRACTIONAL DIFFUSION EQUATION

S. Seminara, M.I. Troparevsky, M. Fabio y G. La Mura

ESTIMACIÓN DEL COEFICIENTE DEL TÉRMINO DIFUSIVO EN UNA ECUACIÓN PARABÓLICA COMPLETA

G.F. Umbricht y D. Rubio

OPTIMIZANDO LA FUSIÓN DE DATOS PARA LA ESTIMACIÓN DE LA DISTRIBUCIÓN DEL TAMAÑO DE PARTÍCULAS. I. ANÁLISIS DEL CRIMEN INVERSO PARAMÉTRICO

F. Otero y G. Frontini 
ECUACIÓN DE BINET EN RELATIVIDAD ESPECIAL

G.R. Chacón . . 335

ROTACIONES DEL $N$-PÉNDULO PARAMÉTRICO DE PLANO INCLINADO CON MIRAS A LA EXTRACCIÓN DE ENERGÍA AMBIENTAL

F.E. Dotti y J.N. Virla

CÁLCULO DEL SEGUNDO COEFICIENTE DE LYAPUNOV USANDO UN MÉTODO EN FRECUENCIA

J.L. Moiola, F.S. Gentile y G.R. Itovich

BIOMATEMÁTICA

SPARSE CONNECTED GRAPHS FOR METRIC SPACES

J.M. Alonso 349

ANÁLISIS TOPOLÓGICO DE AMINOÁCIDOS UTILIZADOS PARA MEJORAR LA SOLUBILIDAD MEDIANTE FORMACIÓN DE COMPLEJOS DE INCLUSIÓN: ESTUDIO PRELIMINAR COLABORATIVO ENTRE LA UNPSJB Y LA UNC

A.V. Dan Córdoba, M.R. Longhi y M.O. Castillo 353

ANÁLISIS DE UN MODELO PARA NEURONAS DEL HIPOCAMPO CON DISTINTOS NIVELES DE RUIDO: INTERACCIÓN ENTRE LA DINÁMICA INTRÍNSECA Y EL RUIDO

U. Chialva y H.G. Rotstein 357

MODELADO DE LA DISTRIBUCIÓN POR SEXO DE PARÁSITOS GEOHELMINTOS Y SU PROBABILIDAD DE APAREAMIENTO

G.M. Lopez y J.P. Aparicio .361

INTERACCIONES DE FILTRO DE FRECUENCIA EN REDES DE NEURONAS NO OSCILATORIAS

A.L. Bel y H.G. Rotstein .365

EL DOMINIO C2B DE LA SINAPTOTAGMINA-1 ES UN REGULADOR CLAVE DE LA ESTABILIZACIÓN DE LOS POROS DE FUSIÓN

M. Caparotta, C.N. Tomes, L.S. Mayorga y D. Masone .369

INTERACCIÓN DE MECANISMOS SEGREGADOS DE RESONANCIA EN CA1-Pyramidal neurons

U. Chialva y H.G. Rotstein .373

EFECTOS DE LOS PROCESOS HOMEOSTÁTICOS EN CIRCUITOS NEURONALES MÍNIMOS

M.V. Ibarra, H.G. Rotstein y G.R. Soto 377

CIENCIA DE DATOS Y APRENDIZAJE AUTOMÁTICO 381

COULD AFFINITY PROPAGATION bioFUEL PROTEIN-PROTEIN INTERACTIONS?

N. Villagra, H. Álvarez, R. Silva y G. Soto .383 
BRIEF EXPLORATORY AND PREDICTIVE ANALYSES OF CONCRETE PROPERTIES USING STANDARD STATISTICAL LIBRARIES AND A MACHINE LEARNING ALGORITHM

H. Alves da Silveira Monteiro y R.L. da Silva Pitangueira 387

BRAINS NEED MATH: OPEN CHALLENGES AT THE INTERSECTION OF AI AND NEUROSCIENCE

G. Kreiman 391

SPARSE ESTIMATION OF THE PRECISION MATRIX AND PLUG-IN PRINCIPLE FOR HYPERSPECTRAL IMAGE CLASSIFICATION

M.L. Picco y M.S. Ruiz

VOX EXPERTORUM: APRENDIZAJE NO SUPERVISADO DE ENSAMBLES DE CLASIFICADORES BINARIOS

G. Stolovitzky

UN AUTOENCODER BASADO EN EL EQUILIBRIO DE WARDROP PARA LA UBICACIÓN ÓPTIMA DE SENSORES DE TRÁFICO

N. Jares, D. Fernandez, P.A. Lotito y L.A. Parente

EVALUATION OF MACHINE LEARNING ALGORITHMS -K NEAREST NEIGHBORS AND SUPPORT VECTOR MACHINES- FOR STRAWBERRIES CLASSIFICATION DURING FOOD DRYING PROCESS

J. Gamboa-Santos y L.A. Campañone $\ldots \ldots \ldots \ldots \ldots \ldots \ldots \ldots \ldots \ldots \ldots \ldots \ldots \ldots \ldots \ldots \ldots \ldots \ldots$. 407

ECONOMÍA MATEMÁTICA $\ldots \ldots \ldots \ldots \ldots \ldots \ldots \ldots \ldots \ldots \ldots \ldots \ldots \ldots \ldots \ldots \ldots \ldots \ldots \ldots \ldots \ldots \ldots \ldots$

FUZZY GROUP IDENTIFICATION PROBLEMS

F. Fioravanti y F.A. Tohmé

LATTICE STRUCTURE OF THE RANDOM STABLE SET IN MANY-TO-MANY MATCHING MARKETS

N. Juárez, P. Neme y J. Oviedo

ON STRONG AND WEAK CORE IN MATCHING MARKETS WITH INDIFFERENCES

N. Juarez y J. Oviedo

(NON-)CONVERGENCE TO STABILITY IN COALITION FORMATION GAMES

A.G. Bonifacio, E. Inarra y P. Neme

ALL SEQUENTIAL ALLOTMENT RULES ARE OBVIOUSLY STRATEGY-PROOF

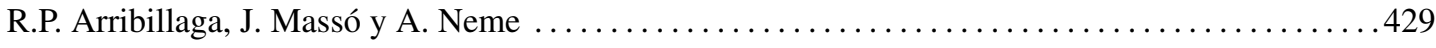

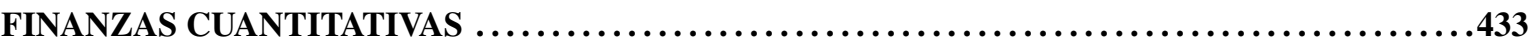

VECTOR ERROR CORRECTION MODEL FOR THE SWAP SPREAD CURVE

E. Ravasi y N.P. Kisbye

A PARAMETRIC CLOSE-FORM APPROXIMATION FOR EUROPEAN MORTGAGE OPTIONS

M. Lopez Galvan 
MODELO DE SALTOS PARA TRAYECTORIAS DE EQUITY Y VALUACIÓN DE OPCIONES

P.M. Gechidjian

NUMERICAL METHODS FOR PRICING OPTIONS WITH TRANSACTION COSTS

F.G. Vega, R. Gonzalez Sosa y J.D. Aguilar

DECOMPOSING THE VIX INDEX INTO GREED AND FEAR

J.A. Serur, J.P. Dapena y J.R. Siri

DESCUBRIMIENTO NO SUPERVISADO Y ANÁLISIS ESTADÍSTICO DE PATRONES DE VELAS PARA PRONOSTICAR RETORNOS FUTUROS DE ACTIVOS FINANCIEROS

G. Falcucci

DELTA HEDGING WITH TRANSACTION COSTS: DYNAMIC MULTI-SCALE STRATEGY USING NEURAL NETS

G. Mazzei, F. Bellora y J. Serur

APLICACIÓN DE REDES NEURONALES LSTM AL PROBLEMA DE PREDICCIÓN DE LA DIRECCIÓN DE MOVIMIENTO DIARIO PARA BITCOIN

A. Muñoz González y F. Salazar Guillen

PROSPECTIVE COMPARISON OF DEEP-LEARNING VS MONTE CARLO METHODS IN HIGHDIMENSIONAL DERIVATIVE PRICING

F. Glancszpigel, F. Bonfanti, G. Michalski y P. Macri

OPTIMAL MARKET MAKING BY REINFORCEMENT LEARNING

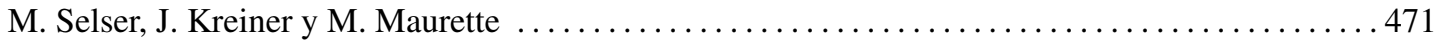

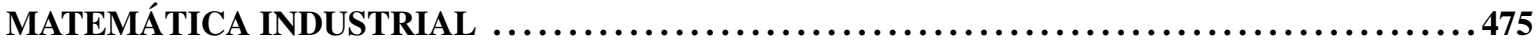

APLICACIÓN DE MÉTODOS DE CONTINUACIÓN NUMÉRICA AL CÁLCULO DE HIPER-LÍNEAS DE EQUILIBRIO TERMODINÁMICO ENTRE FASES INVOLUCRANDO FASES FLUIDAS Y SOLUCIONES SÓLIDAS

A.F. Porras Giraldo, S.B. Rodríguez-Reartes y M.S. Zabaloy 477

CÓMPUTO DE HIPER-LÍNEAS DE EQUILIBRIO MULTIFÁSICO MULTICOMPONENTE REACTIVO

M.J. Molina, S.B. Rodríguez-Reartes y M.S. Zabaloy 481

PROGRAMACIÓN INTEGRADA DE LA PRODUCCIÓN Y LA DISTRIBUCIÓN EN UNA PLANTA BATCH MULTIPRODUCTO

A.S. Tibaldo, Y. Fumero y J.M. Montagna 485

APLICACIÓN INDUSTRIAL DE MODELOS NUMÉRICOS DE EXTRUSIÓN DE ALUMINIO

J.M. Torres Zanardi, A. Scarabino, F. Bacchi y L. Principi .......................... 489

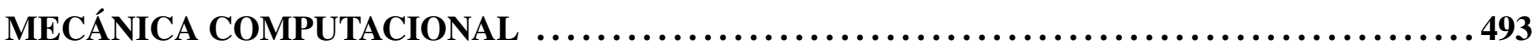

ANÁLISIS PRELIMINAR DEL FLUJO A TRAVÉS DE UNA VÁLVULA DE ALIVIO MEDIANTE EL SOFTWARE OPENFOAM

F.P. Inzeo, H.G. Castro y M.A. Storti 
A PRELIMINARY STUDY OF THE SEPARATION BUBBLE STRUCTURE OVER A SEMI-INFINITE BLUNT BODY

W.R. Miranda, A.L.T. Rezende y N. Ferreira Fico

ANÁLISIS COMPUTACIONAL DE LA SENSIBILIDAD HEMODINÁMICA DE LA POSICIÓN DE UN STENT

L. Telesco, N. Dazeo, A.P. Narata e I. Larrabide

ESTIMACIÓN DE LA VARIACIÓN DE TEMPERATURA DE UN SISTEMA CERRADO MEDIANTE LA SIMULACIÓN DEL MEDIO POR PARTÍCULAS EN MOVIMIENTO BROWNIANO

N.M. Salva

UVLM-ORIENTED MESH GENERATOR FOR WIND TURBINES

B.A. Roccia, L.R. Ceballos, M.L. Verstraete y G. Dimitriadis

DESARROLLO DE UNA PLATAFORMA DE SIMULACIÓN AERODINÁMICA PARA ESTUDIAR GRANJAS DE TURBINAS EÓLICAS

L.R. Ceballos, B.A. Roccia, M.L. Verstraete y S. Preidikman

ESQUEMA DE ACOPLAMIENTO ESTRUCTURAL-AERODINÁMICO BASADO EN FUNCIONES DE BASE RADIAL

L.M. Nitardi, M.L. Verstraete, L.R. Ceballos y B.A. Roccia

DETERMINACIÓN ANALÍTICA DE "BANDGAPS” EN UNA CLASE DE MATERIALES LOCALMENTE RESONANTES

V.H. Cortínez y P.N. Domínguez

DISEÑO DE MECANISMOS FLEXIBLES TIP-TILT PISTON UTILIZANDO TEORÍA DE HELICOIDES

M.A. Pucheta, A.G. Gallardo, R.T. González, J.A. Bernad y S. Fantín

METAMATERIALES MECÁNICOS CON ACTUADORES ELECTROMAGNÉTICOS

M.A. Pucheta, A.G. Gallardo, S. Fantín y R.T. González

MECÁNICA DEL CONTINUO

535

EVALUACIÓN NUMÉRICA DE MAPAS DE POINCARÉ

S. Elaskar, E. del Río y D. Lorenzón 537

SIMULACIÓN NUMÉRICA DEL FLUJO ALREDEDOR DE UN PERFIL AERODINÁMICO EN RÉGIMEN TRANSÓNICO

L. Monaldi y S.A. Elaskar .541

CÁLCULO DE LA ENERGÍA LIBERADA POR LA EXPLOSIÓN DE LA PRIMERA BOMBA ATÓMICA HACIENDO USO DE LA TEORÍA DEL ANÁLISIS DIMENSIONAL

M.J. Frías y S.A. Elaskar .545

CONSIDERACIONES SOBRE LA NUEVA TEORÍA DE INTERMITENCIA CAÓTICA

S. Elaskar y E. del Río 549 
ESTUDIO NUMÉRICO DE UNA SONDA DE LANGMUIR CILÍNDRICA USANDO UN ESQUEMA DE VOLÚMENES FINITOS

D. Lorenzon, S. Elaskar y J.P. Saldia 553

FORMULACIÓN DE UN MODELO DE VIGA PIEZOELÉCTRICA DE TIMOSHENKO INCLUYENDO ALABEO POR TORSIÓN

E. Beltramo, B.A. Roccia, M.E. Pérez Segura y S. Preidikman

IMPLEMENTACIÓN DE UN MÉTODO DE VORTICIDAD DISTRIBUIDA PARA PERFILES QUE CAMBIAN DE FORMA

S. Ribero, A. Aichino, M. Valdez y S. Preidikman 561

IMPLEMENTACIÓN COMPUTACIONAL Y VALIDACIÓN DEL MÉTODO DE RED DE VÓRTICES PARA EL ESTUDIO AERODINÁMICO DE PERFILES ALARES NO DELGADOS

M.F. Valdez, S. Ribero y S. Preidikman .565

UN CONCEPTO NO CONVENCIONAL BASADO EN "FLUTTER" PARA COSECHAR ENERGÍA. PARTE 1: ESQUEMA DE SIMULACIÓN NUMÉRICA

M.E. Pérez Segura, E. Beltramo, B.A. Roccia, M.F. Valdez, M.L. Verstraete, L.R. Ceballos y S.

Preidikman

UN CONCEPTO NO CONVENCIONAL BASADO EN "FLUTTER” PARA COSECHAR ENERGÍA. PARTE 2: EL DISEÑO CONCEPTUAL

M.E. Pérez Segura, E. Beltramo, B.A. Roccia, M.F. Valdez, M.L. Verstraete, L.R. Ceballos y S.

Preidikman

ESTUDIO AERODINÁMICO DE PERFILES ALARES NO DELGADOS POR MEDIO DEL MÉTODO DE RED DE VÓRTICES

M.F. Valdez, S. Ribero y S. Preidikman . ...

UNA FORMULACIÓN MATEMÁTICA ROBUSTA PARA EL CAMBIO DE FORMA DE PERFILES AERODINÁMICOS

A. Aichino, S. Ribero, M. Valdez y S. Preidikman

ON THE THEORETICAL ASPECTS OF THE HOMOGENEOUS TORSION PROBLEM

B.A. Roccia, F. Mazzone y S. Preidikman

MODELLING THE UNIDIRECTIONAL COMPOSITES DYNAMIC FAILURE BY MEANS OF AN EXPONENTIAL SOFTENING LAW INTO A 2D LATTICE MODEL

M. Braun, I. Iváñez y M.P. Ariza

SIMULACIÓN NUMÉRICA DEL ENSAYO DE TRACCIÓN SIMPLE PARA PROBETAS DELGADAS. ANÁLISIS DEL CASO LÍMITE 3D

C. Careglio, C. García Garino, A. Mirasso, D. Celentano, L. Papeleux y J-P. Ponthot

MODEL REDUCTION BY MEAN-FIELD HOMOGENIZATION IN VISCOELASTIC COMPOSITES

M.I. Idiart, N. Lahellec y P. Suquet 
PLANTEO SEMI-ANALÍTICO DEL PROBLEMA VISCOELASTODINÁMICO PARA MEDIOS POROSOS MULTIFÁSICOS CON MICROESTRUCTURAS

J.C. Barreto, J.L. Mroginski y H.A. Di Rado .................................. 601

MODELOS MATEMÁTICOS INTERDISCIPLINARIOS ..........................605

UN MODELO BINIVEL PARA LA ESTIMACIÓN DE LA DEMANDA DE TRANSPORTE PÚBLICO

V.M. Orlando, P.A. Lotito y N. Bhouri .

APLICACIONES DEL MODELADO MATEMÁTICO A LA GESTIÓN DE LOS ESCURRIMIENTOS PLUVIALES URBANOS - CASO GUARAPUAVA, PR, BRASIL

P.T. Stehli, J.F. Weber y L. Redin Vestena

MODELO MATEMÁTICO PARA UN TRATAMIENTO DE AGUAS INDUSTRIALES VÍA OPTIMIZACIÓN

F.E. Buffo y M.C. Vidal

PREDICCIÓN DEL RUIDO VEHICULAR UTILIZANDO REDES NEURONALES ARTIFICIALES

M.E. Sequeira, V.H. Cortínez y A.P. Azzurro

SIMPLIFICACIÓN DE LA ECUACIÓN DE FAO PENMAN-MONTEITH PARA ESTIMAR LA EVAPOTRANSPIRACIÓN DE REFERENCIA A PARTIR DE MODELOS DE REGRESIÓN LINEAL

S. Gavilán, I. Quignard, P.G. Aceñolaza y J.I. Pastore

MODELADO DE YACIMIENTOS NO CONVENCIONALES APLICANDO EL PROYECTO OPEN POROUS MEDIA

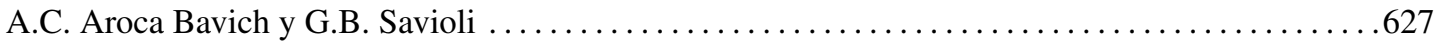

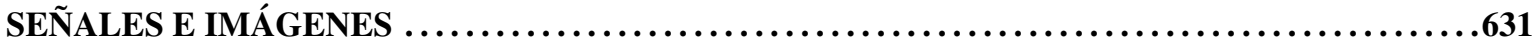

APPLICATION OF ENTROPIC MEASURES IN THE STUDY OF AUDITORY EVOKED POTENTIALS FOR THE DETECTION OF PATHOLOGICAL PATIENTS

M. Baldiviezo, C. Bontempo, J. Barbería, Y. Corsaro, F. Fernandez Biancardi, M. Hernando, M.633 Rodriguez, A. Paglia y W. Legnani

DETECTION OF ATRIAL FIBRILLATION BY ENTROPIC CALCULATION OF ECG SIGNALS

I.G. Ziccardi, P.E. Martínez y W.E. Legnani 637

DISTANCIAS SEMÁNTICAS PARA INFERENCIA EN LA ONTOLOGÍA DE GENES

T. López, D. Milone y L. Di Persia

ESTUDIO DE ESTADOS DE CONSCIENCIA MEDIANTE EL USO DE CUANTIFICADORES DE INFORMACIÓN

N. Fuentes, A. Garcíaz, R. Orofino y D.M. Mateos 645

AN INCREMENTAL VERSION OF SINGULAR SPECTRUM ANALYSIS

J.G. Colonna y B.B. Gatto

CLASIFICACIÓN DE ONDAS LENTAS EN SUEÑO

G. Carbonari, R. Ramele, C. Forcato y M. Pretel 
ANÁLISIS COMPARATIVO DEL DISEÑO AUTOMÁTICO DE W-OPERADORES EN DIFERENTES ESPACIOS COLOR

S.C. Guevara, A. Bouchet, V. Ballarin y J.I. Pastore

CLASIFICACIÓN DE SEÑALES MIOELÉCTRICAS PARA EL CONTROL DE UNA MANO ROBÓTICA

J.M. Fontana, G. Oviedo, R. O’Brien y L. Molisani ........................... 661

TRANSFERENCIA DE CALOR Y MATERIA $\ldots \ldots \ldots \ldots \ldots \ldots \ldots \ldots \ldots \ldots \ldots \ldots \ldots \ldots \ldots \ldots \ldots$

DETERMINACIÓN DE LOS MATERIALES QUE COMPONEN UNA BARRA CON INTERFAZ SÓLIDO-SÓLIDO

G.F. Umbricht, D. Rubio y D.A. Tarzia

TRANSFERENCIA DE CALOR ESTACIONARIA EN MATERIALES MULTICAPA

G.F. Umbricht, D. Rubio y D.A. Tarzia

UN PROBLEMA DE CONDUCCIÓN NO CLÁSICO PARA UNA ECUACIÓN DE DIFUSIÓN FRACCIONARIA

D.N. Goos, S.D. Roscani y D.A. Tarzia

SOLUCIÓN ANALÍTICA DE UN PROBLEMA DE TRANSFERENCIA DE ENERGÍA TÉRMICA CON GENERACIÓN DE CALOR, DISIPACIÓN POR CONVECCIÓN Y FLUJO LATERAL

G.F. Umbricht, D. Rubio y C. El Hasi

VISIÓN COMPUTACIONAL

DETECCIÓN DE ATAQUES DE SUPLANTACIÓN DE IDENTIDAD EN VIDEOS USANDO CÁLCULO DE MOVIMIENTO DEL ROSTRO/FONDO Y REDES NEURONALES

C.I. Orozco y C.A. Martínez

GAZE TRACKING FOR COGNITIVE REHABILITATION

M. Menchón, A. Golimstok y J.M. Massa

SUPERVISED LEARNING FOR SLEEP STAGE SCORING USING RANDOM FOREST: IS A "SIMPLER"MODEL ACCURATE ENOUGH ON UNSEEN INDIVIDUALS?

E. Moris, C. Forcato e I. Larrabide 693

CLASIFICACIÓN DE LA CALIDAD ÓSEA EN IMÁGENES DE DXA UTILIZANDO MACHINE LEARNING

M. Gonzalez, J.M. Massa y N. de Martino

DETECCIÓN DE CAMPTOCORMIA CON MEDICIÓN POSTURAL

G. Carbonari, L. Valenzuela y J. Massa

HERRAMIENTA PARA VISUALIZACIÓN Y SEGMENTACIÓN DE IMÁGENES MÉDICAS BASADA EN WEB

G. Tajes Genga, M. del Fresno y J. D’Amato .705 
PROCESOS DE NACIMIENTOS NO HOMOGÉNEOS Y SU APLICACIÓN A LA PANDEMIA DEL COVID-19

V. Moreno, G. Pena y N. Barraza 711

THE SIR MODEL IN THE COVID-19 PANDEMIC

G. Capobianco, R. Cobiaga, W. Reartes y F. Turpaud

MODELO CON ESTRUCTURA SOCIAL PARA EL ESTUDIO DE MEDIDAS DE CONTROL DE LA PANDEMIA DE COVID-19

M.I. Simoy y J.P. Aparicio 719

DESCONTAMINACIÓN DE BARBIJOS N95 POR MICROONDAS. MODELADO MATEMÁTICO Y SIMULACIÓN COMPUTACIONAL

J.R. Arballo, A.R. Lespinard, S.M. Goñi y R.H. Mascheroni

MOBILITY PATTERN DETECTION FROM COVID-19 DATA WITH TIME VARYING GRAPHICAL LASSO

I.L. Degano y P.A. Lotito

DIFFUSIVE METRICS INDUCED BY MULTIAFFINITIES. THE COVID-19 SETTING FOR BUENOS AIRES (AMBA)

M.F. Acosta, H. Aimar, I. Gómez y F. Morana

MODELO MATEMÁTICO PARA LA EPIDEMIA DE COVID-19

J.G. Neder, P.M. Nuñez, J. Gianatti, P.A. Lotito y L.A. Parente

UN ENFOQUE SIMPLE PARA EL CONTROL DE LA EPIDEMIA DEL COVID-19

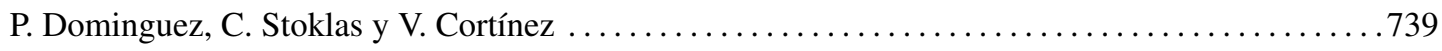

PÓSTERES DE ESTUDIANTES DE GRADO Y POSTGRADO $\ldots \ldots \ldots \ldots \ldots \ldots \ldots \ldots \ldots \ldots \ldots . \ldots 74$

PERSISTENCE AND PERIODIC SOLUTIONS IN SYSTEMS OF DELAY DIFFERENTIAL EQUATIONS

P. Amster y M. Bondorevsky

SIMULACIÓN DE UN MODELO MATEMÁTICO SIMPLIFICADO DE ATEROSCLEROSIS

S.V. Carbonell Berretti y M.M.P. Lucero.

ANÁLISIS DE LA DESPOLARIZACIÓN VENTRICULAR EN EL ELECTROCARDIOGRAMA MEDIANTE LA ENTROPÍA Y COMPLEJIDAD WAVELET

G. Clemente, V. Vampa, E. Valverde y P. Arini 753

MODELADO ESTOCÁSTICO DE UN SISTEMA MAGNÉTICO BICAPA FUERTEMENTE ANISOTRÓPICO

P.S. Pagliaro, G.P. Saracco y M.A. Bab 757 


\section{ECONOMÍA MATEMÁTICA}




\title{
FuZZY GROUP IDENTIFICATION PROBLEMS
}

\author{
Federico Fioravanti ${ }^{\mathrm{a}, \mathrm{b}}$ and Fernando A. Tohmé $\mathrm{e}^{\mathrm{a}, \mathrm{c}}$ \\ anstituto de Matemática de Bahía Blanca, Universidad Nacional del Sur/CONICET, Alem 1253, Bahía Blanca, \\ Argentina \\ ${ }^{\mathrm{b}}$ Departamento de Matemática, Universidad Nacional del Sur, Alem 1253, Bahía Blanca, Argentina \\ ${ }^{\mathrm{c}}$ Departamento de Economía, Universidad Nacional del Sur, San Andrés 800, Bahía Blanca, Argentina
}

\begin{abstract}
We consider a class $N=\{1, \ldots, n\}$ of agents, each one with an opinion about the membership to a group $J$ of the members of the society, consisting in a function $p_{i}: N \rightarrow[0,1]$, indicating for each agent, including herself, the degree of membership to $J$. We consider the problem of aggregating those functions, satisfying different sets of axioms and characterizing different aggregators. While some results are analogous to those of the model with crisp preferences, the fuzzy version is able to overcome some of the main impossibility results found in the literature.
\end{abstract}

Keywords: Group Identification Problems, Fuzzy, Aggregation

2000 AMS Subject Classification: 91B10 - 91B12 - 91B14

\section{INTRODUCTION}

People usually classifies other people, objects or entities in groups. Sometimes these classifications are obvious, as in the assignment of countries to the continent to which they belong. But in many cases, opinions may not be even clear cut and thus it becomes hard to reach a consensus. For instance, if a group of people wants to identify which of them should be considered "tall", finding out whether a member is such may be far from evident. Of course, a consensus could be reached on that people that are 2.00 meters height or more are "tall". But it is not clear whether someone who's height is 1.75 meters can be considered "tall". In this paper we introduce a fuzzy approach to the group identification problem formalized by Kasher and Rubinstein (K-R) in "Who is a J?"[3]. They consider a finite society that has to determine which of its subsets of members consists of exactly those individuals that can be deemed to be $J$ s. By a slight abuse of language, this subset is denoted $J$. Each different set of axioms postulated to yield a solution to this problem characterizes a class of aggregation functions, called Collective Identity Functions (CIFs). The "Liberal" one labels as a $J$ any individual that deems himself to be a $J$; the "Dictatorial" CIF is such that a single individual decides who is a $J$. Finally, the "Oligarchic" CIF determines that somebody is a $J$ if all the members of a given group agree on that.

In our model, every agent, instead of labeling any of the individuals in $N=\{1, \ldots, n\}$ (the society) as belonging or not to $J$, assigns a value in the $[0,1]$ interval to each agent, representing the degree in which that agent is believed to belong to $J$. The axioms in K-R have counterparts in our framework. On the other hand, we also consider another axiom drawn from the literature, namely the Extreme Liberalism axiom [2]. We present fuzzy versions of those crisp axioms. We deal first with the axioms defining the "Liberal" aggregator, showing that the fuzzy versions of the results in K-R remain valid. But when we turn to the axioms defining the Dictatorial aggregator, our results differ from those in the crisp setting. More specifically, K-R prove an impossibility result when the domain and the range of the aggregator are restricted, indicating that the Dictatorial CIF is the only one satisfying those conditions. But there does not exist a clear "translation" of those restrictions into our framework, allowing different interpretations. In some of these we obtain more aggregators verifying the axioms, while in others there does not exist any of them.

The plan of the paper is as follows. Section 2 is devoted to present the model and the set of axioms considered here. In Section 3 we deal with "Liberal" aggregators while in Section 4 we focus on the Dictatorial aggregator. Finally, Section 5 concludes.

\section{MOdEL AND AXIOMS}

Let $N=\{1, \ldots n\}$ be a set of agents that have to define who of them belongs to the group of $J_{\mathrm{s}}$ (which by a slight abuse of language is denoted $J$ ). The opinion of agent $i$ is characterized by a function $p_{i}: N \rightarrow[0,1]$ where $p_{i}(j)$ indicates the assessment of agent $i$ of the degree of membership of $j$ to $J$. 
Agent $i$ has thus a vector of opinions $P_{i}=\left\{p_{i}(1), \ldots, p_{i}(n)\right\}$. A profile of opinions $P$ is a $n \times n$ matrix $P=\left\{P_{1}, \ldots, P_{n}\right\}$. With $\mathbf{P}$ we denote the set of all the profiles of opinions.

A fuzzy subset $J$ of $N$ is characterized by a membership (characteristic) function $f_{J}: N \rightarrow[0,1]$, that indicates the degree of membership of an agent to $J$. Let $\mathbf{F}=\left\{f_{J}: N \rightarrow[0,1]\right\}$ be the set of possible membership functions of a fuzzy subset $J$. We denote by FJ the Fuzzy Collective Identity Function (FCIF) such that $F J: \mathbf{P} \rightarrow \mathbf{F}$.

A FCIF takes a profile of opinions and returns a membership function for the fuzzy subset $J$. More precisely, the membership function of the set $J$ associated to the profile $P \in \mathbf{P}$ is denoted $f_{J}^{P}$. We do not impose further restrictions on this membership function. It can be different for every $i$, for example, $f_{J}^{P}(1)=p_{1}(1)$ while $f_{J}^{P}(2)=\frac{p_{2}(2)}{2}$.

In what follows we will present in an axiomatic way the properties that a social planner would like to see implemented by a "fair" aggregation process, several of them introduced already by K-R in their seminal work.

- Fuzzy Monotonicity (FMON): let $P \in \mathbf{P}$ be such that $f_{J}^{P}(i)=a$ and let $P^{\prime}$ be a profile such that $P_{k, i}^{\prime}>P_{k, i}$ for some $k$ with $P_{h, j}^{\prime}=P_{h, j}$ for all $(h, j) \neq(k, i)$, then $f_{J}^{P^{\prime}}(i) \geq a$.

If $P^{\prime \prime}$ is a profile such that $P_{k, i}^{\prime \prime}<P_{k, i}$ for some $k$ with $P_{h, j}^{\prime \prime}=P_{h, j}$ for all $(h, j) \neq(k, i)$, then $f_{J}^{P^{\prime \prime}}(i) \leq a$.

- Fuzzy Strong Monotonicity (FSMON): let $P \in \mathbf{P}$ be such that $f_{J}^{P}(i)=a$ and let $P^{\prime}$ be a profile such that $P_{k, i}^{\prime}>P_{k, i}$ for some $k$ with $P_{h, j}^{\prime}=P_{h, j}$ for all $(h, j) \neq(k, i)$, then $f_{J}^{P^{\prime}}(i)>a$.

If $P^{\prime \prime}$ is a profile such that $P_{k, i}^{\prime \prime}<P_{k, i}$ for some $k$ with $P_{h, j}^{\prime \prime}=P_{h, j}$ for all $(h, j) \neq(k, i)$, then $f_{J}^{P^{\prime \prime}}(i)<a$.

- Fuzzy Consensus (FC): if $p_{i}(j) \geq a_{j}$ and $p_{i}(j) \leq b_{j}$ for some $a_{j}, b_{j} \in \mathbb{R}, a_{j}, b_{j}>0$ and for all $i \in N$, then $a_{j} \leq f_{J}^{P}(j) \leq b_{j}{ }^{1}$

- Symmetry (SYM): agents $j$ and $k$ are symmetric if:

- $p_{i}(j)=p_{i}(k)$ for all $i \in N-\{j, k\}$

- $p_{j}(i)=p_{k}(i)$ for all $i \in N-\{j, k\}$

- $p_{j}(k)=p_{k}(j)$

- $p_{j}(j)=p_{k}(k)$

Then, if two agents $j$ and $k$ are symmetric it follows that $f_{J}^{P}(j)=f_{J}^{P}(k)$.

- Fuzzy Symmetry (FSYM): agents $j$ and $k$ are fuzzy-symmetric if:

- $p_{i}(j), p_{i}(k) \geq 0.5$ or $p_{i}(j), p_{i}(k)<0.5$ for all $i \in N-\{j, k\}$

- $p_{j}(i), p_{k}(i) \geq 0.5$ or $p_{j}(i), p_{k}(i)<0.5$ for all $i \in N-\{j, k\}$

- $p_{j}(k), p_{k}(j) \geq 0.5$ or $p_{j}(k), p_{k}(j)<0.5$

- $p_{j}(j), p_{k}(k) \geq 0.5$ or $p_{j}(j), p_{k}(k)<0.5$

If two agents $j$ and $k$ are fuzzy-symmetric then $f_{J}^{P}(j), f_{J}^{P}(k) \geq 0.5$ or $f_{J}^{P}(j), f_{J}^{P}(k)<0.5$.

- Independence (I): let $P$ and $P^{\prime}$ be two profiles such that given an agent $j \in N, p_{i}(j)=p_{i}^{\prime}(j)$ for all $i \in N$. Then $f_{J}^{P}(j)=f_{J}^{P^{\prime}}(j)$.

- Fuzzy Independence (FI): let $P$ and $P^{\prime}$ be two profiles such that given an agent $j \in N$ and for every $i \in N$ we have $p_{i}(j) \geq 0.5$ iff $p_{i}^{\prime}(j) \geq 0.5$ and $p_{i}(j)<0.5$ iff $p_{i}^{\prime}(j)<0.5$.

Then $f_{J}^{P}(j) \geq 0.5$ iff $f_{J}^{P^{\prime}}(j) \geq 0.5$.

\footnotetext{
${ }^{1}$ Alcantud and Andrés Calle [1] call this property Unanimity.
} 
- Liberalism (L): if $p_{i}(i)=1$ for some $i \in N$, then $f_{J}^{P}(k)=1$ for some $k \in N$. If $p_{i}(i)=0$ for some $i \in N$, then $f_{J}^{P}(k)=0$ for some $k \in N$.

- Fuzzy Liberalism (FL): if $p_{i}(i) \geq 0.5$ for some $i \in N$, then $f_{J}^{P}(k) \geq 0.5$ for some $k \in N$. If $p_{i}(i)<0.5$ for some $i \in N$, then $f_{J}^{P}(k)<0.5$ for some $k \in N$.

- Extreme Liberalism (EL):

(i) If $p_{i}(j)=1$ for some $i, j \in N$, then $f_{J}^{P}(k)=1$ for some $k \in N$.

(ii) If $p_{i}(j)=0$ for some $i, j \in N$, then $f_{J}^{P}(k)=0$ for some $k \in N$.

\section{- Fuzzy Extreme Liberalism (FEL):}

(i) If $p_{i}(j) \geq 0.5$ for some $i, j \in N$, then $f_{J}^{P}(k) \geq 0.5$ for some $k \in N$.

(ii) If $p_{i}(j)<0.5$ for some $i, j \in N$, then $f_{J}^{P}(k)<0.5$ for some $k \in N$.

\section{LIBERALISM}

We define the Strong Liberal FCIF as:

$$
L\left(P_{1}, \ldots, P_{N}\right)(i)=L_{J}(i)=p_{i}(i)
$$

for all $i \in N$.

Theorem 1 The only FCIF that verifies FMON, FC, FI and FL is the Strong Liberal FCIF.

Corollary 1 The only FCIF that verifies FMON, FC, I and L is the Strong Liberal FCIF.

Corollary 2 If a FCIF verifies FMON, FC, FI and FL then it verifies SYM and FSYM.

Corollary 3 There is no FCIF that verifies FSMON, FC, FI and FL.

We introduce two FCIFs:

- The Unanimity FCIF is defined as: $U\left(P_{1}, \ldots, P_{N}\right)(i)=U_{J}(i)=\min _{j} p_{j}(i)$ for all $i \in N$.

- The Inclusive FCIF is defined as: $\operatorname{Inc}\left(P_{1}, \ldots, P_{N}\right)(i)=\operatorname{Inc}_{J}(i)=\max _{j} p_{j}(i)$ for all $i \in N$. $^{3}$

Theorem 2 Inc is the only FCIF that verifies FMON, FC, FI and EL (i) or FEL (i).

$U$ is the only FCIF that verifies FMON, FC, FI and EL(ii) or FEL(ii).

Corollary 4 There is no FCIF that verifies FMON, FC, FI and FEL or EL.

\section{DiCTATORSHIP}

We define the Dictatorial FCIF with the agent $j$ as a dictator as:

$$
D\left(P_{1}, \ldots, P_{N}\right)(i)=D_{J}(i)=p_{j}(i)
$$

for all $i \in N$.

We propose different restrictions for domain and range of the FCIFs. One possibility is that in a profile $P_{i}$, there exists at least one $j$ such that $p_{i}(j)=1$ and one $k$ such that $p_{i}(k)=0$. We call this set of profiles $\mathbf{P}^{*}$. An alternative set of profiles is $\mathbf{P}^{* *}$, in which for every agent $i$ there exists at least one $k$ and one $j$ such that $p_{i}(k) \geq 0.5$ and $p_{i}(j) \leq 0.5$. Another possible case is that in which $p_{i} \neq \mathbf{1}$ and $p_{i} \neq \mathbf{0}$. We call this set

\footnotetext{
${ }^{2}$ Alcantud and Andrés Calle [1] define this aggregator as the Conjunctive FCIF.

${ }^{3}$ Alcantud and Andrés Calle [1] call this aggregator the Benevolent FCIF.
} 
$\mathbf{P}^{* * *}$.

With respect to the membership functions, we can consider the case in which there exist at least one $j$ and one $k$ such that $f_{J}(j)=1$ and $f_{J}(k)=0$. The class of such function is denoted $\mathbf{F}^{*}$.

We define $\mathbf{F}^{* *}$ as the set of membership functions such that for every profile $P$ there exists at least one $k$ and one $j$ such that $f_{J}^{P}(k) \geq 0.5$ and $f_{J}^{P}(k) \leq 0.5$.

Finally we have the family of functions such that $f_{J} \neq \mathbf{1}$ and $f_{J} \neq \mathbf{0}$. We call this set $\mathbf{F}^{* * *}$.

Theorem 3 Consider FCIFs that satisfy axioms FC and FI.

1. Dictatorial is not the only FCIF such that FJ : $\boldsymbol{P}^{* * *} \rightarrow \boldsymbol{F}^{* * *}$.

2. Dictatorial is the only FCIF such that FJ : $\boldsymbol{P}^{*} \rightarrow \boldsymbol{F}^{* *}$ or FJ $: \boldsymbol{P}^{* *} \rightarrow \boldsymbol{F}^{* *}$, and there is no FCIF such that FJ : $\boldsymbol{P}^{* * *} \rightarrow \boldsymbol{F}^{* *}$.

3. Dictatorial is the only FCIF such that FJ : $\boldsymbol{P}^{*} \rightarrow \boldsymbol{F}^{*}$, and there is no FCIF such that FJ : $\boldsymbol{P}^{* *} \rightarrow \boldsymbol{F}^{*}$ or FJ $: \boldsymbol{P}^{* * *} \rightarrow \boldsymbol{F}^{*}$.

Theorem 4 Consider FCIFs that verify the axioms FC and I.

1. Dictatorial is not the only FCIF such that FJ : $\boldsymbol{P}^{* * *} \rightarrow \boldsymbol{F}^{* * *}$.

2. Dictatorial is the only FCIF such that FJ : $\boldsymbol{P}^{*} \rightarrow \boldsymbol{F}^{* *}$ or FJ: $\boldsymbol{P}^{* *} \rightarrow \boldsymbol{F}^{* *}$, and there is no FCIF such that FJ: $\boldsymbol{P}^{* * *} \rightarrow \boldsymbol{F}^{* *}$.

3. Dictatorial is the only FCIF such that FJ : $\boldsymbol{P}^{*} \rightarrow \boldsymbol{F}^{*}$, and there is no FCIF such that FJ : $\boldsymbol{P}^{* *} \rightarrow \boldsymbol{F}^{*}$ or FJ $: \boldsymbol{P}^{* * *} \rightarrow \boldsymbol{F}^{*}$.

\section{CONCLUSION}

In this work we analyze, from a fuzzy point of view, the Group Identification Problem. The opinions of the agents are no longer crisp statements about the membership or not to a group. Instead of that, their opinions are expressed in terms of degrees of membership to the class of $J$ s. The axioms FL and FEL (L and EL) are very restrictive, and do not allow more rules other than the Strong Liberal FCIF. The binary nature of determining if someone has more or less than 0.5 degree of acceptance, leads in general to the preservation of the uniqueness and impossibility results. However, when we modify the domain and range of the FCIFs, we get the possibility of choosing more rules than just the Dictatorial one.

\section{REFERENCES}

[1] Alcantud, J. C. And de Andrés CAlle, R., The problem of collective identity in a fuzzy environment, Fuzzy Sets and Systems, 315(2017), pp 57-75.

[2] Fioravanti, F. And Tohmé, F., Alternative Axioms in Group Identification Problems, Journal of Classification, 2020, $1-10$.

[3] Kasher, A. And Rubinstein, A., On the Question “Who is a J?": A Social Choice Approach, Logique \& Analyse, 160(1997), pp 385-395. 